Abanico Veterinario. Enero-Diciembre 2021; 11:1-7. http://dx.doi.org/10.21929/abavet2021.15 Artículo Original. Recibido: 29/09/2020. Aceptado: 02/03/2021. Publicado: 20/03/2021. Clave: e2020-81

\title{
Efecto del closantel combinado con fenbendazol sobre huevos de parásitos gastrointestinales en caballos
}

\author{
Effect of closantel combined with fenbendazole on the eggs of gastrointestinal \\ parasites in horses
}

\section{Cerna-Adame Roberto*1 ID, Barrón-Bravo Oscar² ID , Angel-Sahagún Cesar ${ }^{1}$ ID, Hernández-Marín Antonio ${ }^{1}$ ID, Arredondo-Castro Mauricio ${ }^{1 \mathrm{ID}}$, Avila-Ramos Fidel $^{1}$ ID **}

${ }^{1}$ División Ciencias de la Vida, Universidad de Guanajuato, Programa Educativo de Medicina Veterinaria y Zootecnia. ${ }^{2}$ Instituto Nacional de Investigaciones Forestales Agrícolas y Pecuarias, Campo Experimental las Huastecas, Carretera Tampico-Mante $\mathrm{km} \mathrm{55,} \mathrm{Villa} \mathrm{Cuauhtémoc,} \mathrm{Altamira,}$ Tamaulipas, México. CP 89610. *Autor de correspondencia y responsable de la investigación: Fidel Avila Ramos. Departamento de Veterinaria y Zootecnia. Universidad de Guanajuato. División de Ciencias de la Vida, ExHacienda El Copal, Km. 9 Carretera Irapuato-Silao, CP. 36500, Irapuato, Guanajuato, México. b.etobeto@live.com.mx*, barron.oscar@inifap.gob.mx, csahagun@ugto.mx, jahmarin@ugto.mx, arredondo.m@ugto.mx, ledifar@ugto.mx*

\section{RESUMEN}

Los parásitos gastrointestinales deben controlarse periódicamente en los caballos para mantener su rendimiento productivo y adecuado estado de salud. El objetivo de la investigación fue conocer el efecto e inocuidad del closantel combinado con fenbendazol sobre huevos de parásitos gastrointestinales en equinos. La combinación del fármaco se administró a 102 caballos de diferente raza, edad, sexo y peso corporal a dosis de $10 \mathrm{mg}$ por $\mathrm{kg}$ cada fármaco. Las constantes fisiológicas se midieron antes y 90 minutos después de administrado el gel. Adicionalmente, se colectó una muestra de heces directamente al recto de los caballos durante la exploración física para determinar la presencia y cantidad de huevos. En animales positivos se tomaron muestras a los 15, 30 y 45 días posteriores al tratamiento para conocer el tiempo que pueden proteger a los caballos los fármacos administrados. En los resultados 53 caballos fueron positivos a parásitos, el fármaco presentó eficacia del $99 \%$ a los 15 días post tratamiento y del $89.79 \%$ a los 45 días, en los 102 caballos que recibieron el tratamiento no presentaron ningún efecto sobre las variables fisiológicas evaluadas. Se concluye que la combinación de closantel con fenbendazol a $10 \mathrm{mg}$ por $\mathrm{kg}$ de peso corporal tiene eficacia del $99 \%$ post aplicación y no causo efecto negativo para los caballos independientemente del sexo y la edad.

Palabras claves: Equinos, antihelmínticos, endectocidas, reacción adversa, huevos gastrointestinales, inocuidad.

\begin{abstract}
Gastrointestinal parasites should be periodical controlled in horses to maintain their productive performance and adequate health. The objective of the research was to know the effect and safety of closantel combined with fenbendazole on the eggs of gastrointestinal parasites in horses. The drug combination was administered to 102 horses of different breed, age, sex and body weight at a dose of $10 \mathrm{mg}$ per $\mathrm{kg}$ each drug. Physiological constants were measured before and 90 minutes after the drug was administered. Additionally, a sample was collected directly from the rectum of the horses during the physical examination to determine the presence and quantity of eggs. In positive animals, samples were taken 15,30 y 45 days after treatment to determine the time that the administrated drugs can protect the horses. In the results, 53 horses were positive for parasites, the drug showed efficacy of $99 \%$ at 15 days after treatment and 89.79 at 45 days, in the 102 treated horses there was no effect on the physiological variables evaluated. It is concluded that the combination of closantel with fenbendazole at $10 \mathrm{mg}$ per $\mathrm{kg}$ of body weight has efficacy of $99 \%$ post application and is safe for horses.
\end{abstract}

Keywords: Anthelmintics, endectocides, adverse reaction, gastrointestinal eggs, innocuous. 


\section{INTRODUCCIÓN}

Los parásitos internos en los caballos deben controlarse con formulaciones seguras, inocuas y eficientes para mantener su desempeño productivo. La resistencia parasitaria es un problema mundial, los parásitos que afectan a los caballos son resistentes a los principales productos químicos comerciales usados para su control (García et al., 2013). Para aumentar la eficacia de los fármacos y disminuir la cantidad de parásitos se deben buscar alternativas (Kaplan, 2002; Matthews, 2014); una propuesta es combinar principios activos como es el closantel, combinado con el fenbendazole.

El closantel pertenece al grupo de las salicilanidas, con fórmula química $\mathrm{C}_{22} \mathrm{H}_{14} \mathrm{Cl}_{2} \mathrm{I}_{2} \mathrm{~N}_{2} \mathrm{O}_{2}$ y peso molecular de $663.07 \mathrm{~g} / \mathrm{mol}$, su DL50 es de $50 \mathrm{mg} / \mathrm{kg}$ vía IM. Se administra a dosis de 10 a $40 \mathrm{mg} / \mathrm{kg}$ para controlar Strongylus edentatus, Strongylus vulgaris, Triodontophorus spp, Gasterophilus intestinalis y Anaplocephala perfoliata (Gokbulut y McKellar, 2018). En equinos la dosis sugerida es de $10 \mathrm{mg} / \mathrm{Kg}$ por vía oral con 40 días de tiempo de retiro (Guerrero et al., 1983; Guerrero, 1984). En torrente sanguíneo el closantel se une a la albumina y alcanzar su concentración máxima a las 40 horas, su vida media es de tres semanas con biodisponibilidad vía oral del $50 \%$. El compuesto bloquea la fosforilación oxidativa a nivel mitocondrial, evitando la disponibilidad de energía causando la muerte de los parásitos (Hennessy y Ali, 1997).

El fenbendazol es un polvo blanco de nombre químico 5-(feniltio)-1H-benzimidazol-2il ácido carbónico metiléster, peso molecular de $299.34 \mathrm{~g} / \mathrm{mol}$ y su fórmula es $\mathrm{C}_{15} \mathrm{H}_{13} \mathrm{~N}_{3} \mathrm{O}_{2} \mathrm{~S}$ (Hennessy y Ali, 1997). Es usado para controlar parásitos en bovinos, caballos, porcinos, perros y gatos. En equinos está indicado para grandes y pequeños Strongylus spp y Oxyuris equi, se recomienda a dosis de $10 \mathrm{mg} / \mathrm{kg}$ vía oral caballos (Guerrero et al., 1983; Guerrero, 1984). La DL50 en caballos se da al exceder los 10 $\mathrm{g} / \mathrm{kg}$ administrado vía oral, no tiene reportes negativos; sin embargo, pueden presentarse reacciones de hipersensibilidad.

El objetivo de la presente investigación fue conocer el efecto e inocuidad de closantel, combinado con fenbendazol, a dosis de $10 \mathrm{mg} / \mathrm{kg}$ sobre huevos de parásitos gastrointestinales en heces de caballos.

\section{Lugar de estudio}

\section{MATERIAL Y MÉTODOS}

El estudio se realizó en el estado de Guanajuato, México, en el municipio de Irapuato; ubicado a los $101^{\circ} 20^{\prime} 48^{\prime \prime}$ de Longitud Oeste del meridiano de Greenwich, a los $20^{\circ} 40^{\prime} 18$ " de Latitud Norte; y en el municipio de Pénjamo, localizado a los 10142' 22" de Longitud Oeste del Meridiano de Greenwich y a los 20 25' 44" Latitud Norte; ambas localidades a 1,730 m sobre el nivel del mar (INEGI, 2015).

\section{Unidades experimentales}

Se administró closantel combinado con fenbendazol, a dosis de $10 \mathrm{mg} / \mathrm{kg}$ de peso vivo para cada compuesto; su presentación en pasta con aplicador - dosificador para $600 \mathrm{~kg}$ de peso vivo a 102 caballos (Productos Farmacéuticos S.A. de C.V.). Los animales tratados fueron de diferente raza, edad variable de 1 - 30 años y diferente peso corporal, infestados o no con parásitos gastrointestinales de forma natural. El experimento fue supervisado y el protocolo autorizado por el Comité de Investigación AG (OFAG02-2019). 


\section{Examen físico}

A todos los caballos se les aproximó su edad dental, se les realizó un examen físico a través de su frecuencia cardiaca, respiratoria, movimientos peristálticos y tiempo de llenado capilar, antes y 90 minutos posteriores de administrado el fármaco.

\section{Administración del fármaco}

Para administrar la pasta, se calculó el peso vivo del caballo en kilogramos, con el procedimiento morfométrico de Martinson et al. (2014). Posteriormente, se ajustó el aplicador de Vermi-Horse Silver® con el anillo graduador al peso respectivo del caballo. mantenido en ayuno para introducir el aplicador en el espacio interdental. La pasta se colocó en la parte posterior de la lengua, levantando la cabeza del caballo durante cinco segundos para que pueda deglutir el medicamento.

\section{Análisis de laboratorio}

Se colectaron $30 \mathrm{~g}$ de heces obtenidas directamente del recto de los caballos, para examinar la presencia o ausencia de huevos de parásitos gastroentéricos a través del recuento de los huevos en las heces $(\mathrm{RHH})$; a las muestras positivas se les contó la cantidad de huevos de parásitos en las heces, con el procedimiento de la cámara de McMaster. A los caballos positivos se le realizaron tres muestreos consecutivos posteriores a la aplicación del fármaco a los 15, 30 y 45 días.

\section{Análisis estadístico}

La cantidad de huevos encontrados en las heces de los machos se compararon con los encontrados en heces de las hembras, usando la prueba de medias independientes con el estadístico t de Student con el procedimiento PROC TTEST de SAS y nivel de significancia de $\mathrm{P} \leq 0.05$. Para conocer el nivel de infestación y efecto de la localidad, se realizó una correlación de Pearson entre la cantidad de huevos y localidad, usando el procedimiento PROC CORR de SAS a un nivel de significancia de $P \leq 0.05$. Las variables fisiológicas evaluadas antes y después de aplicado el fármaco; así como la cantidad de huevos en las heces a los 15, 30, y 45 días, se compararon con medias pareadas con la prueba $t$ de Student, usando el procedimiento PROC T de SAS. Todos los datos se analizaron con el paquete estadístico SAS (2012).

\section{RESULTADOS}

En la investigación se tomaron muestras a 102 caballos; 53 animales salieron positivos a Strongylus Spp. y dos equinos de los 53 también presentaron huevos de Parascaris Spp., la prevalencia fue del $51.9 \%$. La presencia de huevos en heces fecales disminuyó por efecto del tratamiento $(P \leq 0.05)$; en machos, la eficacia fue del 98.87, 83.56 y $91.68 \%$ y en las hembras del $99.23,93.11$ y $87.90 \%$, a los 15,30 y 45 días (tabla 1), respectivamente. Además, la cantidad de huevos en heces fecales de hembras fue mayor comparada con los machos ( $\mathrm{P} \leq 0.05)$. Los caballos de Irapuato presentaron más huevos (25.52) en las heces fecales ( $\mathrm{P} \leq 0.05)$, comparados con los equinos de Pénjamo (10.58) (tabla 1). La edad de los caballos no presentó ninguna relación con la cantidad de huevos encontrados en las heces fecales (datos no mostrados). 
Tabla 1. Cantidad de huevos de parásitos gastrointestinales en $\mathrm{g}$ de heces en machos $\mathrm{y}$ hembras a los $0,15,30$ y 45 días del experimento $(\mathrm{N}=53)$

\begin{tabular}{|c|c|c|c|c|}
\hline \multirow[t]{2}{*}{ Sexo } & & \multicolumn{2}{|c|}{ Día } & \multirow[b]{2}{*}{45} \\
\hline & 0 & 15 & 30 & \\
\hline $\begin{array}{l}\text { Machos } \\
\text { (31) }\end{array}$ & $14.35^{\mathrm{b}}$ & 0.16 & 2.35 & 1.19 \\
\hline $\mathrm{P}^{*}$ & & 0.0202 & 0.0415 & 0.0211 \\
\hline $\begin{array}{l}\text { Hembras } \\
\text { (22) }\end{array}$ & $29.72^{a}$ & 0.22 & 2.04 & 3.59 \\
\hline $\mathrm{P}^{*}$ & & 0.0096 & 0.0101 & 0.0051 \\
\hline
\end{tabular}

En las variables fisiológicas de los caballos no hubo cambios por efecto del medicamento a los 90 minutos post aplicación (tabla 2).

Tabla 2. Variables fisiológicas de caballos tratados con pasta de closantel-fenbendazol vía oral a los 90 minutos ( $\mathrm{N}=102)$

\begin{tabular}{lccc}
\hline Variable & \multicolumn{2}{c}{ Medición } & Probabilidad \\
Frecuencia cardiaca & Inicial $^{\star}$ & Final $^{\star *}$ & 0.3699 \\
Frecuencia respiratoria & $40.0 \pm 9.6$ & $39.5 \pm 8.1$ & 0.7195 \\
Temperatura corporal & $20.8 \pm 9.4$ & $21.1 \pm 8.0$ & 0.0705 \\
Tiempo de llenado capilar & $37.8 \pm 0.8$ & $37.9 \pm 0.8$ & 0.5663 \\
Movimientos peristálticos & $2.8 \pm 0.6$ & $2.8 \pm 0.5$ & 0.3197 \\
\hline
\end{tabular}

${ }^{*}$ Inicial = antes de la administración del fármaco.

**Final= 90 minutos posteriores a la administración del fármaco.

\section{DISCUSIÓN}

El control de parásitos gastrointestinales en caballos es una actividad común integrada al calendario de manejo, pero no se obtiene un diagnóstico del tipo y cantidad de parásitos de la localidad o el lugar donde se ubica la unidad productiva (García et al., 2013). Bedoya et al. (2011) reportaron prevalencias del 92\% y Cala-Delgado et al. (2016) del $71 \%$, porcentajes altos comparados a los resultados obtenidos en el estudio de 51.9\%, pero son porcentajes cercanos a los reportados por Aromaa et al. (2018) del $57.6 \%$ en diferentes razas y tipos de equinos. La variación en la prevalencia y la cantidad de parásitos encontrados puede ser causada por el fin zootécnico, la localidad, tipo de alimentación, manejo sanitario de los animales y la diversidad de los parásitos en las zonas de estudio. En las investigaciones los géneros más reportados son Trichostrongylus, Trichonema spp. y Strongylus spp (Cala-Delgado et al. (2016). Sin embargo, en el estudio realizado todos los caballos positivos presentaron huevos de Strongylus Spp, y sólo dos caballos también presentaron Parascaris Spp.

El estudio sólo tiene la finalidad de conocer el efecto de la pasta sobre la cantidad de huevos y las especies de parásitos no se consideraron, pero fueron identificadas dos.

En equinos, el control de parásitos gastrointestinales se realiza de manera cotidiana; Buzatu et al. (2015) determinaron prevalencias mayores a 50 huevos de parásitos por gramo de heces en 137 caballos de 195 muestreados. A las 10 semanas posteriores al tratamiento determinaron que los caballos sólo se mantienen protegidos menos de tres meses. Sanna et al. (2016) usaron lactonas macrocíclicas y benzimidazoles para evaluar su efecto del día 0 hasta los 150 días posteriores a su aplicación. Los 
resultados obtenidos para eliminar huevos de parásitos totales fueron del $99.7 \%$ y a los 14 días del 99\%. Resultados similares muestra el estudio presentado con la combinación propuesta a la dosis indicada por el fabricante.

El principio activo usado puede determinar la eficiencia del tratamiento y tiempo de protección en los caballos. En tratamiento de elección son fármacos en pasta con ivermectina, pamoato de pirantel, moxidectina con praziquantel; o su combinación ivermectina-praziquantel. Caffe et al. (2018) evaluaron ivermectina a dosis de $0.2 \%$ $\mathrm{mg} / \mathrm{kg}$ como tratamiento de elección con eficacia para reducir el conteo fecal de huevos del $99.8 \%$ en yeguas y $100 \%$ en potrancas a los 15 días, resultados similares a los encontrados en el presente estudio combinando closantel-fenbendazol. En ningún caballo se observó efecto de la edad en los muestreos sobre la cantidad de huevos en heces; los caballos más jóvenes tenían un de 1.5 a 2 años de vida y los más grandes 30 años, aproximadamente.

En caballos de carga o de tiro la incidencia parasitaria es mayor, comparados con animales usados para deportes, entretenimiento o terapias. Tedla y Abichu (2018) determinaron $72.2 \%$ de incidencia en animales de carga, porcentajes mayores a las encontradas en nuestro estudio, debido al ambiente de trabajo y tipo de cuidado que reciben. En las cuadras, lienzos y agrupamientos policiacos los animales tienen cuidados periódicos, esto significa que tienen programas de medicina preventiva establecidos de forma general. Pero los caballos destinados a trabajo de campo no reciben los mismos cuidados, su desgaste metabólico es mayor debido a sus condiciones de vida generales. Por lo tanto, es común encontrar resultados que superan a los reportados en el presente estudio, debido al deficiente manejo, condiciones de trabajo y su estado nutricional; así como el pastoreo que realizan en los potreros o lugares de trabajo, edad de los hospedadores, e incluso la raza y sexo pueden afectar los resultados de los estudios (Caffe et al., 2018).

El sexo de los caballos puede ser un factor predisponente para describir y reportar los resultados en las investigaciones de los equidos. Bedoya et al., (2011) reportan que el sexo del caballo no tiene efecto sobre la incidencia de los parásitos; pero nuestros resultados indican que las hembras tienen $48 \%$ más huevos de parásitos por gramo de heces fecales, comparadas con los machos; hallazgos evidentes que pueden sugerir que la cantidad de huevos de parásitos pueden estar relacionado con el sexo de los equinos.

\section{CONCLUSIÓN}

La eficacia encontrada en el estudio confirma que el closantel combinado con el fenbendazol a $10 \mathrm{mg}$ por $\mathrm{kg}$ de cada fármaco es del $99.05 \%$ a los 15 días, y su administración es segura para los caballos.

\section{AGRADECIMIENTOS}

Los autores agradecen el apoyo recibido de la empresa de Productos Farmacéuticos S.A. de C.V. Al Lienzo charro RZL de Irapuato, Lienzo Charro de Pénjamo, a la Secretaría de Seguridad Pública Policía Montada de Irapuato, a la MVZ. Katherin Estefani Maya Ramírez y todos los particulares que facilitaron sus caballos para realizar la investigación. 


\section{LITERATURA CITADA}

AROMAA M, Hautala K, Oksanen A, Sukura A, Näreaho A. 2018. Parasite infections and their risk factors in foals and young horses in Finland. Veterinary Parasitology: Regional Studies and Reports. 12: 35-38. https://doi.org/10.1016/j.vprsr.2018.01.006

BEDOYA MA, Arcila VH, Díaz DA, Reyes EA. 2011. Prevalencia de parásitos gastrointestinales en équidos del municipio de Oiba (Santander). Spei Domus. 7(15): 17-23. ISSN: 1794-7928. https://revistas.ucc.edu.co/index.Php/sp /is sue/view/71

BUZATU MC, Mitrea IL, Miron L, Lonita M. 2015. Efficacy of two anthelmintic products on strongyles in horses from stud farms in Romania. Agriculture and Agricultural Science Procedia. 6: 293 -298. https://doi.org/10.1016/j.aaspro.2015.08.075

CAFFE G, Paz B, Cerutti J, Cooper L, Signorini M, Anziani O. 2018. Resistencia antihelmíntica en equinos. Observaciones sobre el período de reaparición de huevos luego del tratamiento con ivermectina en potrancas y yeguas madres. Revista Fave. (17): 40-44. https://doi.org/10.14409/favecv.v17i2.7660

CALA-DELGADO DL, Santos-Parra ME, Ortiz-Pineda L, Yunis-Aguinaga J. 2016. Prevalencia de parásitos gastrointestinales en équidos del municipio de Coromoro (Santander, Colombia). Spei Domus. 12(25): 1-6. https://doi.org/10.16925/23824247.2016.02.02

GARCÍA A, Brady HA, Nichols WT, Prien S. 2013. Equine Cyathostomin resistance to fenbendazole in Texas horse facilities. Journal of Equine Veterinary Science. 33: 223228. https://doi.org/10.1016/j.jevs.2012.06.005

GOKBULUT C, McKellar QA. 2018. Anthelmintic drugs used in equine species. Veterinary Parasitology. 33(4): 223-228. https://doi.org/10.1016/j.vetpar.2018.08.002

GUERRERO J, Michael BF, Rohovsky MW, Campbell BP. 1983. The activity of closantel as an equine antiparasitic agent. Veterinary Parasitology. 12(1): 71-77.

https://doi.org/10.1016/0304-4017(83)90090-0

GUERRERO J. 1984. Closantel: A review of its antiparasitic activity. Preventive Veterinary Medicine. 2(1-4): 317-327. https://doi.org/10.1016/0167-5877(84)90075-8

HENNESSY DR, Ali DN. 1997. The effect of feed intake level on the pharmacokinetic disposition of closantel in sheep. International Journal for Parasitology. 27(9): 10811086. https://doi.org/10.1016/S0020-7519(97)00076-3

INEGI (Instituto Nacional de Estadística y Geografía). 2015. Estadísticas Irapuato, Guanajuato. México. https://www.inegi.org.mx/app/areasgeograficas/?ag=11

KAPLAN RM. 2002. Anthelmintic resistance in nematodes of horses. Veterinary Research. 33: 491-507. https://doi.org/10.1051/vetres:2002035 
MARTINSON KL, Coleman RC, Rendahl AK, Fang Z, McCue MA. 2014. Estimation of body weight and development of a body weight score for adult equids using morphometric measurements. Journal of Animal Science. 92: 2230-2238.

https://doi.org/10.2527/jas.2013-6689

MATTHEWS JB. 2014. Anthelmintic resistance in equine nematodes. International Journal Parasitol Drugs Drug Resistance. 4(3): 310-315.

https://doi.org/10.1016/j.ijpddr.2014.10.003

SAS institute. 2012. Statistical Analysis Sofware SAS/STAT®. version 9.0.2. Cary, N.C., USA: SAS Institute Inc., ISBN: 978-1-60764-599-3.

https://www.sas.com/en_us/software/stat.html

SANNA G, Pipia A, Tamponi C, Manca R, Varcasia A, Traversa D, Scala A. 2016. Anthelmintics efficacy against intestinal Strongyles in horses of Sardinia, Italy. Parasite Epidemiology and Control. 1(2): 15-19. https://doi.org/10.1016/j.parepi.2016 .01.001

TEDLA M, Abichu B. 2018. Cross-sectional study on gastro-intestinal parasites of equids in south-western Ethiopia. Parasite Epidemiology and Control. 3(4): 1-6. https://doi.org/10.1016/j.parepi.2018.e00076 